\title{
HIGH ANGULAR RESOLUTION OBSERVATIONS OF HER 36 *
}

\author{
B. STECKLUM \\ $M P G$ working group "Dust in star-forming regions", Jena, FRG \\ R.R. HOWELL \\ University of Wyoming, Laramie, U.S.A. \\ A. ECKART \\ Max Planck Institute for Extraterrestrial Physics, Garching, FRG \\ and \\ A. RICHICHI \\ Max Planck Institute for Astronomy, Heidelberg, FRG
}

\begin{abstract}
The star Herschel 36 (Her 36) which ionizes most of the Hourglass nebula has been observed during a lunar occultation as well as by speckle interferometry in the near infrared (NIR). From the lunar occultation data the strip brightness profile has been extracted which shows that in the L' band Her 36 is extended with a FWHM of 0.45 ". Subsequent speckle interferometry revealed 11 sources in a $6 " x$ " 6 " area at a level of $0.15^{\prime \prime}$ resolution. From the comparison of the brightness profile obtained by the occultation and the strip brightness profile synthesized from the speckle image, we conclude that Her 36 is surrounded by a very young infrared cluster.
\end{abstract}

Key words: NIR - speckle observations - lunar occultation - Her 36 - star formation

\section{Observations}

The lunar occultation observation has been performed using the $2.3 \mathrm{~m}$ telescope of WIRO on May 19th, 1992. The reappearance of the source has been measured in the L' band using the diaphragm of 4" diameter and the integration time of $2 \mathrm{~ms}$. The final source position has been acquired before the disappearance by finding the maximum flux near the nominal position. At the time of the reappearance slight cirrus was present. A check of the position after the event showed that tracking the position of the maximum signal has been successfully maintained.

The $\mathrm{K}$ band speckle imaging of Her 36 has been carried out using the SHARP camera (Eckart et al. 1991) at ESO's NTT on August 12th, 1992. One quadrant of the detector chip (128x128 pixels) has been used for imaging at the scale of 50 mas/pixel. Altogether 2200 images of Her 36 at the integration time of $0.5 \mathrm{~s}$ have been collected. A corresponding number of reference frames was taken by using the nearby $9 \mathrm{Sgr}$. The average seeing was about 0.5 " as estimated from the speckle frames.

\section{Results}

The light curve of the reappearance of Her 36 is shown in Fig.1. From the rise time of the signal and the absence of a diffraction pattern, it is obvious that the source must be extended. For the retrieval of the strip brightness profile, two different algorithms have been applied. The first one is a model-independent procedure implemented by Richichi (1989). As the second, a regularization algorithm has been used which

* Based on observations collected at the European Southern Observatory, La Silla, Chile. 


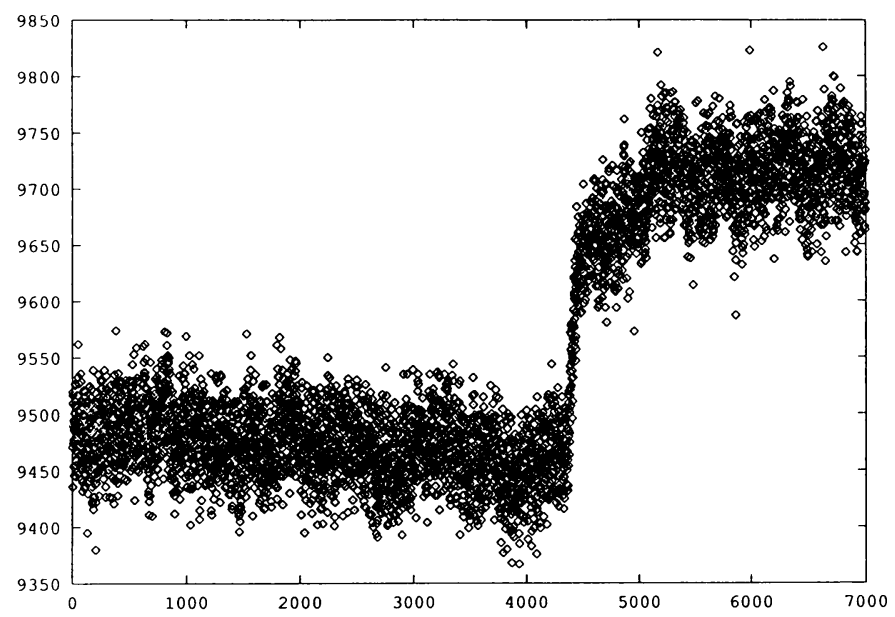

Fig. 1. Reappearance of Her 36. The data have been binned to $10 \mathrm{~ms}$.

incorporates singular value decomposition. Despite the latter lacks of the positivity constraint, the features of the object profiles are in good agreement. The FWHM of the strip brightness profile of Her 36 is 0.45 ". The secondary event in the light curve (about $6 \mathrm{~s}$ after the reappearance of Her 36 ) is caused by the binary KS1. It puts constraints on the location of the diaphragm in respect to the speckle frame. The NIR speckle data have been stacked after correction for dead pixels and flat fielding by the shift-and-add method. The resulting image has been processed by Lucy deconvolution as well as by phase calibration using $9 \mathrm{Sgr}$ as reference. The Lucy deconvolved image has been reconvolved with the 0.15 " beam and is given as a contour plot in Fig.2. From the $\mathrm{K}$ band imaging, the presence of 11 sources in the field has been established. Their positions (relative to Her 36) and magnitudes are given in the table. The magnitudes are based on the value of $7.5^{\mathrm{m}}$ for Her 36 from Woodward et al. (1990) which includes the flux of the components $1,4,6$, and 7 . The source KS1 of Woodward et al. (1990) consists of a binary star. The coordinate differences (") are positive for positions east and north of Her 36, respectively.

\begin{tabular}{rrrrl}
\hline No. & $\mathrm{K}$ & $\Delta \alpha$ & $\Delta \delta$ & Remarks \\
\hline 1 & 7.56 & 0.0 & 0.0 & Herschel 36 \\
2 & 9.8 & 0.45 & 3.45 & KS1 primary \\
3 & 11.0 & 0.55 & 2.90 & KS1 secondary \\
4 & 11.2 & -0.25 & -0.67 & \\
5 & 11.5 & 2.50 & -1.66 & \\
6 & 12.5 & 0.60 & 0.15 & \\
7 & 12.8 & 0.25 & -0.61 & multiple? \\
8 & 13.7 & 2.23 & -0.93 & \\
9 & 13.7 & -1.12 & 0.14 & \\
10 & 14.1 & -1.13 & 1.14 & \\
11 & 14.8 & 3.13 & -0.06 & \\
\hline
\end{tabular}




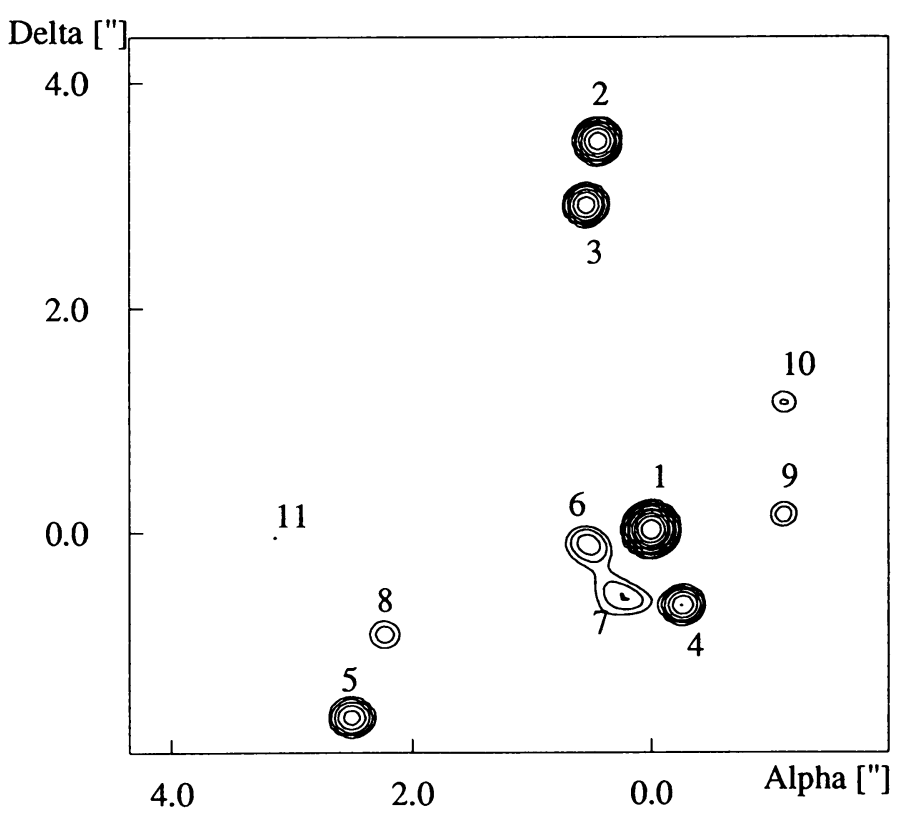

Fig. 2. Contour plot of the Her 36 region. Objects are labeled according to Tab.1. Contours range from $0.1 \%$ to $100 \%$ in steps of 2 .

The brightness profile of Her 36 may be explained by the integration over the nearby sources parallel to the lunar limb (position angle of Her 36 was $237^{\circ}$ ), assuming that their relative contribution to the total L' flux is larger than at $\mathrm{K}$. Therefore, it is likely that the $\mathrm{K}-\mathrm{L}$ ' index of these objects exceeds that of Her 36 (K-L'=1.2 mag) and is similar to $\mathrm{L}^{-\mathrm{K}^{\prime}}=2.2 \mathrm{mag}$ of $\mathrm{KS} 1$.

From the speckle imaging, it can be ruled out that KS1 is a dust knot as proposed by Woodward et al. (1990). The sources surrounding Her 36 seem to form a very young compact star cluster probably containing pre-main-sequence objects. There is no evidence from the NIR imaging for a disk-like structure around Her 36 which has been claimed by Lightfoot et al. (1984).

\section{Acknowledgements}

B. Stecklum acknowledges a travel grant from the DFG (Ste 605/2).

\section{References}

Eckart, A., Hofmann, R., Duhoux, P., Genzel, R. and Drapatz, S.: 1991, ESO Messenger 65, 1 Lightfoot, J.F., Deighton, D.W., Furniss, I., Glencross, W.M., Hirst, C.J.,Jennings, R.E. and Poulter, G.: 1984, Mon.Not.R.ast.Soc 208, 197

Richichi, A.: 1989, Astron.Astrophys. 226, 366

Woodward, C.E., Pipher, J.L., Helfer, H.L. and Forrest, W.J.: 1990, Astrophys.J. 365, 252 